\title{
SOCIOLOGIE QUANTITATIVE : QUELQUES REPÈRES MÉTHODOLOGIQUES À L'USAGE DE L'ENQUÊTE EN MILIEU ECCLÉSIAL
}

Christophe Monnot

Institut protestant de théologie | «Études théologiques et religieuses »

2018/4 Tome 93 | pages 543 à 557

ISSN 0014-2239

Article disponible en ligne à l'adresse :

https://www.cairn.info/revue-etudes-theologiques-etreligieuses-2018-4-page-543.htm

Distribution électronique Cairn.info pour Institut protestant de théologie.

(C) Institut protestant de théologie. Tous droits réservés pour tous pays.

La reproduction ou représentation de cet article, notamment par photocopie, n'est autorisée que dans les limites des conditions générales d'utilisation du site ou, le cas échéant, des conditions générales de la licence souscrite par votre établissement. Toute autre reproduction ou représentation, en tout ou partie, sous quelque forme et de quelque manière que ce soit, est interdite sauf accord préalable et écrit de l'éditeur, en dehors des cas prévus par la législation en vigueur en France. Il est précisé que son stockage dans une base de données est également interdit. 


\title{
Sociologie quantitative : quelques repères méthodologiques à l'usage de l'enquête en milieu ecclésial
}

\begin{abstract}
Christophe MONNOT* fait état des compétences nécessaires pour préparer une enquête quantitative dans les milieux religieux et paroissiaux. Il traite aussi du protocole d'enquête fondé sur des hypothèses scientifiques et des critères de fiabilité, ainsi que des méthodes d'administration d'un questionnaire, de l'échantillonnage et de la collecte des données.
\end{abstract}

Les transformations sociales actuelles ne manquent pas de marquer le milieu ecclésial. Cette situation ne cesse d'intriguer les sciences sociales comme les théologiens ${ }^{1}$ (et les différents acteurs religieux) qui y ont tout d'abord vu une privatisation du religieux ${ }^{2}$, puis une forme de mort de Dieu ${ }^{3}$ pour ensuite parler

\footnotetext{
* Christophe Monnot est maître de conférences en sociologie des protestantismes à la Faculté de théologie protestante de l'université de Strasbourg et chercheur FNS Senior à l'université de Lausanne. La méthodologie développée par Christophe Monnot met en lumière que toute enquête s'appuie sur un matériau dont le choix et la sélection constituent eux aussi des présupposés, légitimes dans la mesure où ils sont identifiés et explicités. Ce que fait l'auteur lorsqu'il signale que l'enquête porte sur les paroisses catholiques du canton suisse de Fribourg. Sa contribution montre également que toute analyse d'une situation donnée s'effectue à partir d' « un centre de perspective » particulier (nous empruntons l'expression, utilisée en référence aux travaux de Gérard Genette, à Oswald Ducrot, Le dire et le dit, Paris, Minuit, 1984, p. 208). Ce concept est particulièrement utile en méthodologie dans la mesure où il permet de distinguer les différents points de vue qui s'expriment dans une même enquête. Les intérêts de l'institution qui est à l'origine de l'enquête, le statut du chercheur qui la mène, de même que les résultats de recherche ne se recoupent pas : ils peuvent parfois confirmer mais doivent tout autant pouvoir contredire les postulats du chercheur ainsi que les attentes de l'institution (Note de l'éditeur, F. Moser).

${ }^{1}$ Relevons par exemple deux ouvrages emblématiques d'Harvey Cox sur ce sujet : Harvey G. Cox, La cité séculière : essai théologique sur la sécularisation et l'urbanisation, Tournai, Casterman, 1968 ; ID., Retour de Dieu : voyage en pays pentecôtiste, Paris, Desclée de Brouwer, 1995.

${ }^{2}$ Voir Roland J. CAMPICHE et al., Croire en Suisse(s) : analyse des résultats de l'enquête menée en 1988/1989 sur la religion des Suisses, Lausanne, L'Âge d'homme, 1992.

${ }^{3}$ Voir Steve Bruce, God is Dead: Secularization in the West, Oxford, Blackwell, 2002.
} 
d'un retour du religieux ${ }^{4}$ ou d'une révolution spirituelle ${ }^{5}$. C'est dans ce débat que l'enquête quantitative occupe une place de choix. Elle fournit une partie importante des données nécessaires pour comprendre ces transformations.

Pourtant, ne nous y trompons pas. La production de mesures, de chiffres et de données statistiques, aussi séduisante qu'elle puisse paraître, ne débouche pas nécessairement sur les éclairages envisagés ou sur des résultats à potentiel scientifique. C'est d'ailleurs en réaction à une modélisation statistique du religieux, pensée exclusivement en termes de changement de pratique collective, que le sociologue Thomas Luckmann avait, dans les années 1960, écrit sur la « religion invisible ${ }^{6} »$. Mais depuis Luckmann, les transformations n'ont cessé de se multiplier au point que la religion collective de son époque est devenue invisible, et que la religion invisible qu'il voulait interroger est devenue bien plus manifeste.

Ces transformations posent de nouvelles questions et poussent les Églises à se restructurer et à se repenser continuellement ${ }^{7}$. La pression est mise pour une part sur la théologie pratique qui doit continuellement évoluer avec ces changements $^{8}$. La tentation est donc forte de lancer une étude pour obtenir des données chiffrées. Pour en tirer pleinement profit, il est nécessaire d'en connaître les écueils.

Cette contribution a pour objectif d'anticiper les principaux pièges, en rappelant le schéma global d'une enquête et les différentes étapes d'un sondage quantitatif, afin de s'assurer de la représentativité des mesures et des chiffres obtenus. Nous abordons ici cinq étapes essentielles à la mise en place d'une enquête que nous illustrons par une enquête quantitative menée auprès des paroisses catholiques de la ville de Fribourg 9 .

\footnotetext{
${ }^{4}$ Voir Peter Ludwig Berger (éd.), The Desecularization of the World: Resurgent Religion and World Politics, Grand Rapids, Eerdmans, 1999.

${ }^{5}$ Voir Paul Heelas, Linda Woodhead (éd.), The Spiritual Revolution: Why Religion is Giving Way to Spirituality, Londres, Blackwell, 2005.

${ }^{6}$ Thomas Luckmann, The Invisible Religion: the Problem of Religion in Modern Society, Londres, Collier-Macmillan, 1967. La première version allemande datant de 1963 a pour titre le sous-titre de la version anglaise.

${ }^{7}$ Voir Jörg Stolz, Edmée BALlif, L’avenir des Réformés : les Églises face aux changements sociaux, Genève, Labor et Fides, 2011.

${ }^{8}$ Olivier BAUER, Le protestantisme et ses cultes désertés : lettres à Maurice qui rêve quand même d'y participer, Genève, Labor et Fides, 2008 ; Élisabeth PARMENTIER, Les filles prodigues : défis des théologies féministes, Genève, Labor et Fides, 1999.

${ }^{9}$ Christophe MonNot, François-Xavier AMHERDT, « Horaires de messes et promesses pastorales », Lumen Vitae 70/4 (2015), p. 445-466.
} 


\section{LE PROTOCOLE D’ENQUÊTE}

Le but principal d'une enquête quantitative est le recueil de données chiffrées dans un temps relativement court pour en produire une analyse représentative d'une population ${ }^{10}$. Afin de générer des données fiables, elle doit pouvoir $\mathrm{s}^{\prime}$ intégrer à un cadre théorique solide ${ }^{11}$. Pour défendre ce principe essentiel, les chercheurs allemands en médecine Thomas Höfer, Hildegard Przyrembel et Silvia Verleger ont publié en 2004 un article sur les « nouvelles évidences de la théorie des cigognes ${ }^{12}$ » dans lequel ils montrent que la baisse des cigognes dans la Basse-Saxe est corrélée à la baisse des naissances dans cette région. De plus, l'augmentation de la population des cigognes aux alentours de Berlin est corrélée à l'augmentation des naissances en dehors des hôpitaux de la ville. Des statistiques, des tableaux et diverses données viennent soutenir de manière convaincante, et dans un langage scientifique, cette théorie des cigognes. La visée des auteurs à travers cette publication, au demeurant sérieuse, est bien sûr de démontrer que la théorie qui est à la base d'une analyse peut conduire à dire à peu près n'importe quoi ! Les cigognes n'ont absolument rien à voir avec les naissances, simplement il se trouve que l'urbanisation et le développement industriel d'une région font fuir les cigognes et influent sur le taux de natalité. Il s'agit donc de deux conséquences distinctes issues de causes complexes qui se trouvent ici et pour diverses raisons corrélées. Cet exemple vient souligner le besoin crucial d'une théorie solide et d'hypothèses validées scientifiquement avant de se lancer dans une enquête ou bien une analyse de données.

Une enquête est donc partie prenante d'un processus de recherche scientifique plus vaste. Elle est menée dans l'objectif de répondre à des questions ou des intrigues discutées dans le champ académique ${ }^{13}$. La définition d'une problématique constitue la première étape importante dans la mise en place d'une recherche quantitative. Il est alors nécessaire de se questionner également sur les enjeux sociaux et académiques de l'enquête, ses spécificités, ainsi que sur ses modalités avant de se lancer. Cette question n'est pas triviale dans le champ religieux, car le sentier institutionnel qu'a suivi telle ou telle organisation ecclésiale, sa hiérarchie, ses valeurs et ses croyances peuvent anéantir les perspectives ouvertes par la mise en place d'une recherche. L'enquête quantitative est donc inscrite entre deux

\footnotetext{
${ }^{10}$ Frédéric LEBARON, L'enquête quantitative en sciences sociales : recueil et analyse de données, Paris, Dunod, 2006.

${ }^{11}$ Nous recommandons au chercheur intéressé par la réalisation d'une enquête de lire : Howard S. BECKER, Les ficelles du métier : comment conduire sa recherche en sciences sociales, Paris, La Découverte, 2002.

${ }^{12}$ Thomas Höfer, Hildegard Przyrembel, Silvia Verleger, « New evidence for the Theory of the Stork », Paediatric and Perinatal Epidemiology 18/1 (2004), p. 88-92. 2005.

${ }^{13}$ Olivier Martin, L'enquête et ses méthodes : l'analyse quantitative de données, Paris, Armand Colin,
} 
pôles : le débat scientifique général et le débat spécifique au sein de l'institution qui soutient le projet.

Une fois assuré que l'enquête est réalisable autant scientifiquement qu'institutionnellement, l'enquêteur peut passer à la phase suivante, qui consiste à mettre en place un protocole global d'enquête. Ce protocole souligne la marche à suivre, ainsi que les potentiels écueils théoriques, sociaux et pratiques qui pourraient contrevenir à son bon déroulement ${ }^{14}$. Les différentes étapes d'une enquête doivent être placées sur une ligne chronologique afin d'être en adéquation avec la problématique traitée et la population à investiguer.

On peut résumer un protocole d'enquête en dix étapes réunies en un premier tableau et dont nous reprenons les principaux éléments plus en aval pour les discuter.

Tableau 1 - Dix étapes du protocole de recherche

Étapes du protocole d'enquête

Définition de l'objet de recherche

Détermination des principales

hypothèses et identification des mesures à effectuer

Sélection d'une population de référence à sonder

Choix d'un mode de récolte de données

Construction d'un questionnaire

Constitution d'un échantillon

Collecte de données

Production d'une base de données

Analyse statistique

Communication des résultats

\section{Descriptif}

Que cherche-t-on à investiguer?

Quelle problématique cherche-t-on à éclairer?

Hypothèses théoriques à tester.

Comment les mesurer de manière valide?

Public représentatif de la problématique posée

Méthode la plus en adéquation avec le public et la problématique pour obtenir des données Choix des questions, des modalités de réponse, pré-test des questions, etc.

Sélection d'une population représentative de la population à investiguer

Administration concrète de l'enquête auprès du public

Nettoyage des données, tests de validité interne, codage et recodage des variables obtenues à partir du questionnaire

Confrontation des hypothèses avec les données recueillies, modélisation

Communication auprès du public des résultats généraux, production de tableaux simples, présentations et contributions scientifiques

\footnotetext{
${ }^{14}$ Nous avons montré ailleurs que, par exemple, les différentes manières d'évaluer la pratique religieuse avaient une incidence sur les taux obtenus. Voir Christophe MonNOT, « Mesurer la pratique religieuse. Différentes mesures, différents taux ? Analyse comparative à partir de la Suisse », Archives de sciences sociales des religions 158/1 (2012), p. 137-156.
} 
Ce tableau permet de sérier également les écueils potentiels ainsi que le temps nécessaire à la mise en place d'une enquête. La partie publique de l'administration d'un questionnaire ne représente en fait qu'une petite étape dans un long processus d'enquête. Afin d'illustrer le propos, je prendrai appui sur une enquête que j'ai menée en collaboration avec François-Xavier Amherdt, professeur en théologie pratique à l'université de Fribourg ${ }^{15}$.

Avant de me solliciter, les responsables spirituels des paroisses et des unités pastorales de la ville de Fribourg et des environs se sont entendus sur la finalité de l'enquête. Il leur semblait opportun de recueillir l'avis des fidèles sur les différents types de messes proposés. Comptant 69 messes dominicales pour moins de 25000 membres, l'idée était alors de déterritorialiser la réflexion et de pouvoir s'appuyer sur une autre approche offrant des types de messes davantage en rapport avec les publics différenciés (familles, jeunes, personnes âgées, etc.). Les responsables avaient donc pour ambition de recueillir l'avis des membres afin de proposer des messes variées avec des horaires adaptés aux différentes composantes de la population catholique locale. Après que le projet ait mûri en 2011-2012, j'ai été contacté pour produire un questionnaire d'enquête et des analyses. Le sondage a eu lieu en juin 2013 et les résultats ont été présentés publiquement en février 2014. Ces quelques balises chronologiques permettent de souligner que si le sondage se passe dans un temps relativement court - un week-end dans ce cas -, la préparation de l'enquête, de même que le travail d'analyse jusqu'à la publication des résultats, est pour sa part assez long et demande une réflexion importante.

\section{FIABILITÉ de L'ENQUÊTE}

Une fois la finalité déterminée, il faut s'assurer de la fiabilité de l'enquête. Une enquête quantitative ne va pas de soi. Toutes les étapes du protocole sont motivées par le souci d'approcher la meilleure fiabilité possible. À cette fin, les enquêteurs doivent s'assurer de maximiser l'adhésion des répondants. Par adhésion, on entend la participation en pleine conscience des tenants et des aboutissants de l'enquête de la part des répondants. Le type de questionnaire doit alors permettre, à partir du fond et de la forme, de la susciter ${ }^{16}$. Deux niveaux sont à considérer comme l'indique le tableau ci-après.

\footnotetext{
${ }^{15}$ Chr. Monnot, F.-X. AmHerdT, « Horaires de messes et promesses pastorales », art. cit.

${ }^{16}$ François de SingLY, L'enquête et ses méthodes : le questionnaire, Paris, Nathan, 1992.
} 
Tableau 2 : Deux niveaux d'adhésion du questionnaire

\begin{tabular}{|l|l|l|}
\hline Niveau & Pour l'enquêteur & Pour le répondant \\
\hline Cognitif & $\begin{array}{l}\text { Définir des réponses } \\
\text { simples, abordables et } \\
\text { compréhensibles }\end{array}$ & $\begin{array}{l}\text { Les questions complexes, } \\
\text { demandant de se concentrer, } \\
\text { de se remémorer des } \\
\text { événements, fatiguent le } \\
\text { répondant et facilitent la } \\
\text { non-réponse }\end{array}$ \\
\hline Social & $\begin{array}{l}\text { Chercher des répondants } \\
\text { volontaires, fiables et } \\
\text { décrivant la réalité telle } \\
\text { qu'ils la conçoivent }\end{array}$ & $\begin{array}{l}\text { Socialement, le répondant, } \\
\text { par sa volonté de répondre, } \\
\text { peut chercher à plaire par } \\
\text { ses réponses (désirabilité } \\
\text { sociale), ne pas divulguer } \\
\text { son avis ou rester mesuré } \\
\text { dans ses opinions }\end{array}$ \\
\hline
\end{tabular}

Ces données indiquent que le premier niveau d'adhésion serait de type cognitif. Il s'intéresse à ce que les questions soient abordables et compréhensibles. Les questions qui demandent trop de réflexion risquent de perdre le répondant et de le décourager de continuer l'enquête. Les batteries de questions faisant appel à des souvenirs, à des situations passées ou à des connaissances spécifiques poussent le répondant à des activités cognitives trop importantes pour un temps si court, poussant à l'hésitation, l'approximation ou à l'évitement de la question. C'est pourquoi il est utile de restreindre le nombre de ce type de questions et de les répartir parcimonieusement dans le questionnaire.

Le deuxième niveau est d'ordre social. Dans une enquête, il est indispensable de s'assurer que le répondant est volontaire et désireux de participer au sondage. Cette volonté peut aussi cacher une désirabilité sociale. C'est-à-dire qu'en répondant, la personne cherche d'abord à se montrer désirable plutôt qu'à donner son point de vue, son opinion ou son évaluation concrète de la réalité qu'il perçoit. Il est tout aussi décisif de soigner les questions abordant des sujets sensibles, afin d'éviter que le répondant ne se sente jugé, évalué ou comparé.

Dans l'enquête menée à Fribourg, sur le plan cognitif, une question sur les différents types de messes incluait la mesure d'opinion favorable à la messe en latin. Cette question a suscité un vaste débat au sein du groupe de pilotage. Certains soulignaient que théologiquement parlant, on la qualifierait de « forme extraordinaire ", tandis que d'autres considéraient que les fidèles comprendraient mieux le terme de «messe en latin ». Cependant, que ce soit l'une ou l'autre des 
formulations, toutes deux comportent une ambiguité dans la mesure où les messes ordinaires peuvent comporter des séquences (chants, récitations, prières...) ellesmêmes en latin. Pourtant, la finalité de l'enquête étant de mesurer l'appréciation des différentes formes de messes, il n'était pas question de la supprimer. Il fallut donc trouver une formulation simple et compréhensible pour tous.

Au sujet de la désirabilité sociale, une question cruciale concernait les célébrations dominicales en l'absence de prêtre. Pour le comité de pilotage, cette question revêtait un enjeu particulier, puisqu'il craignait que l'enquête ne soit interprétée comme une volonté de sonder l'avis des membres sur ce seul sujet. En effet, avec la crise des vocations, l'actualité catholique pousse de plus en plus de paroisses à célébrer la messe en l'absence d'un ecclésiastique (quand bien même cette question ne se trouve pas inscrite à l'agenda de la région de Fribourg). Dans cette situation, il fallait alors faire un choix entre la finalité mesurer l'opinion sur les différents types de messes - et le risque de froisser le répondant. Il a été décidé de maintenir la question, mais de la placer à la fin de cette batterie de questions sur les types de messes. On l'observe, il n'y a pas de question anodine dans un questionnaire et chacune doit être évaluée en fonction de la finalité de l'enquête, mais aussi des interprétations possibles de la part d'une partie des répondants.

\section{LE MODE D'ADMINISTRATION DE L'ENQUÊTE}

Du point de vue méthodologique, l'obsession de l'enquêteur est de s'assurer de la fiabilité de la recherche qu'il engage ${ }^{17}$. Pour garantir la fiabilité, le mode d'administration du questionnaire est une pièce primordiale du puzzle, même si l'enquête ne se résume pas à un questionnaire. Quatre modes d'administration sont disponibles : le questionnaire en face à face $(\mathrm{QFF})$, le questionnaire autoadministré (QAA), le questionnaire par téléphone (Computed-assisted telephone interviewing, CATI) et le questionnaire sur Internet (Computed-assisted web interviewing, CAWI). Une fois le mode d'administration déterminé se pose le problème de la longueur du questionnaire, sachant que plus un questionnaire est long, plus on récolte d'informations. Et néanmoins, plus un questionnaire est long, plus il est coûteux, tant financièrement qu'en matière de concentration pour le répondant, qui peut alors se lasser et répondre avec moins d'attention, ou même être tenté d'abandonner.

Le troisième tableau liste ces différents modes d'administration en soulignant leurs avantages et leurs inconvénients. Parfois ce sont des critères

\footnotetext{
${ }^{17}$ Nicole BERTHIER, Les techniques d'enquête en sciences sociales : méthodes et exercices corrigés, Paris, Armand Colin, 1998.
} 
économiques qui vont guider la sélection du mode d'administration d'un questionnaire, parfois des spécificités propres à l'enquête, comme la récolte de données très précises par un questionnaire en face à face.

Tableau 3 : Comparaison entre quatre modes d'administration d'un questionnaire

\begin{tabular}{|c|c|c|}
\hline Mode & Avantages & Inconvénients \\
\hline $\begin{array}{l}\text { CAPI : questionnaire en face } \\
\text { à face assisté d'un ordinateur }\end{array}$ & $\begin{array}{l}\text { Permet les images, } \\
\text { les dessins, etc. } \\
\text { Moins d'erreurs } \\
\text { d'interprétation } \\
\text { Améliore la participation }\end{array}$ & $\begin{array}{l}\text { Coût élevé } \\
\text { Difficulté à fixer un rendez- } \\
\text { vous } \\
\text { Effets d'influence (de } \\
\text { l'enquêteur sur le répondant) } \\
\text { et biais de la désirabilité } \\
\text { sociale du répondant } \\
\text { Difficultés relatives aux } \\
\text { données sensibles }\end{array}$ \\
\hline $\begin{array}{l}\text { QAA : questionnaire auto- } \\
\text { administré }\end{array}$ & $\begin{array}{l}\text { Faible coût } \\
\text { Sentiment d'anonymat } \\
\text { renforcé } \\
\text { Liberté de répondre selon son } \\
\text { agenda } \\
\text { Informations spécifiques }\end{array}$ & $\begin{array}{l}\text { Nécessité d'un questionnaire } \\
\text { bref, clair et précis } \\
\text { Risque d'incompréhension } \\
\text { Facilite la non-réponse } \\
\text { partielle (saut de questions) } \\
\text { et totale (non renvoi du } \\
\text { questionnaire) }\end{array}$ \\
\hline $\begin{array}{l}\text { CATI : questionnaire par } \\
\text { téléphone }\end{array}$ & $\begin{array}{l}\text { Faible coût } \\
\text { Effet de neutralité par rapport } \\
\text { à l'enquête } \\
\text { Autorise des questionnaires } \\
\text { plus longs } \\
\text { Permet des explications }\end{array}$ & $\begin{array}{l}\text { Refus plus massif } \\
\text { Difficulté de joindre } \\
\text { le correspondant } \\
\text { De plus en plus de contacts } \\
\text { sont hors annuaire } \\
\text { Risque de rupture } \\
\text { de conversation lorsque } \\
\text { le questionnaire est long } \\
\text { Nécessité d'une formulation } \\
\text { très bien structurée des } \\
\text { questions }\end{array}$ \\
\hline $\begin{array}{l}\text { CAWI : questionnaire } \\
\text { par Internet }\end{array}$ & $\begin{array}{l}\text { Faible coût } \\
\text { Avantages identiques à } \\
\text { la collecte auto-administrée } \\
\text { Rapidité de la collecte et } \\
\text { de la constitution de la base } \\
\text { de données } \\
\text { Relances possibles } \\
\text { Peut toucher un grand } \\
\text { nombre de personnes }\end{array}$ & $\begin{array}{l}\text { Beaucoup de non-réponses } \\
\text { Beaucoup d'abandons après } \\
\text { quelques réponses } \\
\text { Peu adapté aux } \\
\text { questionnaires longs } \\
\text { Biais de la sélection des } \\
\text { répondants (favorables } \\
\text { à l'enquête ou ayant des } \\
\text { compétences spécifiques) }\end{array}$ \\
\hline
\end{tabular}


Si le mode d'administration en QFF permet une enquête plus détaillée avec moins d'erreurs d'interprétation des questions, son coût reste élevé. Elle favorise la désirabilité sociale, dans la mesure où le répondant est davantage tenté de répondre pour plaire à l'enquêteur (ce dernier pouvant également l'influencer). Ce mode d'administration peut également être assisté d'un ordinateur par l'enquêteur.

Le QAA a l'avantage d'être d'un plus faible coût, puisque c'est le répondant qui remplit lui-même les réponses, renforçant ainsi le sentiment d'anonymat. Les questionnaires doivent cependant être courts et extrêmement clairs, à défaut de quoi, l'enquêteur encourt le risque d'un désintérêt du répondant tenté de sauter certaines des questions ou de ne pas renvoyer le questionnaire.

Le sondage par CATI est peu coûteux. Il permet aussi une plus grande neutralité des échanges entre l'enquêteur et le répondant et autorise des questionnaires plus longs. Le désavantage est qu'il devient de plus en plus difficile de joindre les personnes (la vogue des sondages publicitaires ayant incité les gens à ne pas ou ne plus figurer sur les annuaires) et que lorsque l'on atteint un répondant potentiel, on doit encore faire face à un fort taux de refus.

L'enquête par Internet - le CAWI - semble cumuler les avantages du QAA et du CATI et bénéficie d'une facilité technique permettant d'atteindre un grand nombre de personnes. Ce type d'enquête a cependant le désavantage de se heurter à de nombreux abandons après quelques questions, et de ne pas pouvoir garantir la représentativité de la population ciblée puisque seul un certain type de population répond via l'Internet (population sachant manier l'outil informatique et numérique).

Lors de l'enquête que nous avons menée à Fribourg, la question du mode d'administration s'est posée en ces termes. Comme la finalité de l'enquête était de recueillir l'opinion des membres des paroisses, il était utile d'investiguer prioritairement les usagers des célébrations dominicales et, en second lieu, les membres des différents conseils. Le mode d'administration le plus simple (et le plus rapide) fut celui du questionnaire auto-administré, qui permet de cocher et d'inscrire des réponses rapidement tout en maintenant l'anonymat. Il s'est avéré commode pour les paroissiens de pouvoir répondre sur une feuille reçue au cours de la messe, de pouvoir la remplir sur les bancs de l'église, ou, pour les responsables, lors d'une séance de conseil. Ce mode d'administration a eu cependant un impact sur la taille du questionnaire. Dans le cas de notre exemple, une contingence pratique a déterminé l'ampleur du sondage : il fallait impérativement ne pas dépasser quatre pages de format $\mathrm{A} 4$, de façon à obtenir une page $\mathrm{A} 3$, recto-verso, pliable en deux, facilement imprimable dans la plupart des paroisses, ne nécessitant aucun agrafage et n'impliquant aucune feuille volante (qui pourrait se perdre). 
Le comité de pilotage a décidé qu'il ferait passer les questionnaires pendant une célébration dominicale (en lieu et place de l'homélie). On distribua les questionnaires avec un crayon, puis on le releva une dizaine de minutes plus tard. Une personne compta les personnes présentes et nota le nombre de questionnaires reçus en retour (afin de calculer le taux de réponse). La procédure d'administration fut plus aisée pour les conseils regroupant seulement une cinquantaine de personnes concernées.

Pour obtenir un avis représentatif d'une population paroissiale active, il était important de mener le sondage un dimanche ordinaire. Un week-end a été choisi pour ces raisons. Ensuite, une équipe de bénévoles a été formée et préparée pour pouvoir imprimer dans chaque paroisse les questionnaires, s'assurer des conditions matérielles, organiser une distribution rapide ainsi qu'une collecte efficace des formulaires complétés. Cet exemple montre que du choix du mode d'administration de l'enquête découle l'organisation et l'infrastructure de l'enquête. Une décision élémentaire telle que le choix d'un mode de passation a des incidences pratiques et concrètes sur la longueur du questionnaire et la manière de le faire parvenir aux sondés. Il résulte un savant dosage entre quantités de données, moyens d'atteindre aisément les répondants, coûts et faisabilité concrète de l'opération.

\section{UNE POPULATION REPRÉSENTATIVE}

Pour la fiabilité d'une enquête, un autre point d'attention particulier est la sélection de la population des personnes que l'on désire sonder. Pour différentes raisons, notamment de coût et de faisabilité, on ne cherche pas à atteindre une population dans son intégralité. On opère plutôt par échantillonnage représentatif d'une population. L'échantillon peut être défini comme la population de référence en modèle réduit. Il s'agit donc, tout d'abord, de bien la circonscrire. La définition déterminera les paramètres d'exclusion et d'inclusion dans l'échantillon. Elle conditionnera également les critères de représentativité.

Deux grandes méthodes d'échantillonnage sont habituellement suivies en sciences sociales, la méthode aléatoire et celle des quotas. Le quatrième tableau offre une vue synoptique et comparative de ces deux méthodes. 
Tableau 4 : Méthodes d'échantillonnage

\begin{tabular}{|l|l|}
\hline Échantillonnage aléatoire & Échantillonnage par méthode des quotas \\
\hline $\begin{array}{l}\text { Nécessite un fichier exhaustif de la } \\
\text { population de référence }\end{array}$ & $\begin{array}{l}\text { Nécessite des critères de choix } \\
\text { S'avère plus simple et moins couteux }\end{array}$ \\
\hline Risque de non-réponse & On réitère jusqu'à ce que le quota soit atteint \\
\hline Grande souplesse dans la sélection & $\begin{array}{l}\text { Doit connaitre les critères de représentativité } \\
\text { de la population avant d'être retenu }\end{array}$ \\
\hline Grande fiabilité des estimations & $\begin{array}{l}\text { Possible biais dû à de mauvais critères de } \\
\text { départ }\end{array}$ \\
\hline $\begin{array}{l}\text { Des données parfois manquantes pour un } \\
\text { type particulier de population }\end{array}$ & $\begin{array}{l}\text { Possible biais dû à des populations plus } \\
\text { volontaires qui ont rempli le quota }\end{array}$ \\
\hline Plus adapté aux grands échantillons $>1000$ & Plus adapté aux petits échantillons $<100$ \\
\hline
\end{tabular}

Dans les grandes enquêtes de sociologie, on mélange fréquemment les deux types d'échantillonnage, en usant de quotas pour certaines tranches de la population, en stratifiant la population et en surreprésentant une partie minoritaire, afin d'avoir assez d'individus pour l'analyse.

La taille minimale d'un échantillon est importante à définir pour avoir une représentativité statistique. Des formules simples sont dans ce cas appliquées, prenant en compte la taille de la population de référence, la fréquence du phénomène, la précision souhaitée. Il est cependant contre-indiqué de mener des calculs statistiques sur un nombre de personnes inférieur à trente ${ }^{18}$.

Dans le cas de l'enquête quantitative sur les paroisses de Fribourg, la question de la population a été abordée de la manière suivante. Premièrement, il a fallu choisir une population cible. Parmi les catholiques affiliés, allait-on sonder des paroisses de la ville et des environs en utilisant le fichier d'adresses mis à jour par l'Église (via l'administration municipale) ? Allait-on sonder seulement ceux qui participent régulièrement à la vie des paroisses en procédant alors à un sondage au cours d'une messe ordinaire ? Allait-on questionner uniquement les membres ayant des responsabilités particulières (conseil

\footnotetext{
18 À ce propos, lisez les notices de tests de produits de beauté : les effectifs n'atteignent jamais les trente personnes, ce qui permet d'offrir des pourcentages très aguicheurs, mais qui statistiquement se différencient peu du hasard.
} 
d'Unité pastorale, conseil de la communauté ecclésiastique, conseil de paroisse, conseil pastoral, etc.)? Comme évoqué précédemment, c'est la population paroissiale qui a été sélectionnée par le comité de pilotage, au motif qu'elle représentait le cœur des opinions nécessaires à investiguer pour envisager une restructuration des messes ${ }^{19}$.

Une seconde question surgit d'emblée, celle de l'accord de l'ensemble des paroisses et des entités ecclésiales pour participer à l'enquête. Deux facteurs pouvant potentiellement fragiliser l'accord de principe ont fait l'objet d'une attention particulière : les minorités nationales et les responsables des communautés ecclésiastiques - entité importante qui gère l'aspect financier incluant les impôts ecclésiastiques.

Au sujet des minorités nationales, en dehors des messes données dans les deux langues (français et allemand) parlées au sein de la ville, Fribourg compte cinq missions linguistiques catholiques. Fonctionnant comme des paroisses indépendantes les unes des autres, les paroisses linguistiques portugaise, croate, vietnamienne, italienne et espagnole furent toutes sollicitées pour participer à l'enquête. Composées de primo-arrivants (ou secondos), les populations des minorités nationales sont, pour différentes raisons, encore moins enclines à répondre à un sondage ${ }^{20}$, tandis que ces cinq missions participent de manière notable à la vitalité de la pratique avec des fidèles plus jeunes que les autres paroisses en ville. Afin d'éviter l'écueil de la non-participation, deux stratégies ont été suivies ici. Premièrement, traduire le questionnaire dans les langues principales des missions linguistiques, et deuxièmement expliquer clairement aux responsables des missions les enjeux de l'enquête et les avantages qu'ils auraient à y participer. Après une première séance de présentation assez crispée, un terrain d'entente a pu être trouvé et une deuxième séance a permis de convaincre les responsables du bien-fondé de leur participation. Un taux de réponse important a pu être ainsi atteint, ce qui représente un résultat exceptionnel et singulier en Suisse dans le cadre d'une enquête quantitative auprès de ce type de population. En conséquence, les spécificités des missions linguistiques ont pu apparaître dans les résultats. Ces derniers ont permis de souligner les demandes particulières d'une population plus jeune et très active du catholicisme fribourgeois.

Du côté des responsables des communautés ecclésiastiques, l'impact n'est pas à négliger, car il suffit à quelques conseillers, souvent également engagés dans les instances politiques locales, d'activer leur réseau politique pour dénoncer des « manœuvres » et décourager les fidèles de participer. Dans le

\footnotetext{
${ }^{19}$ Voir à ce propos Nancy T. Ammerman, Pillars of Faith: American Congregations and their Partners, Berkeley, University of California Press, 2005 ; ID, Congregation and Community, NouveauBrunswick, Rutgers University Press, 1997.

${ }^{20}$ Voir Francesco LAGANÀ et al., « National minorities and their representation in social surveys: which practices make a difference? », Quality \& Quantity 47/3 (2013), p. 1287-1314.
} 
cas de cette enquête, des délégués ont collaboré, en amont, à sa mise en place. Des questionnaires assortis d'une lettre d'introduction spécifique ont été préparés à leur intention. Ils offraient la possibilité de comparer leurs réponses avec les besoins des populations de fidèles. Bien qu'au final aucune différence significative n'ait été observée, la participation des conseillers a permis de créer une atmosphère de confiance pour l'administration auprès des membres réguliers.

\section{LA COLLECTE DES DONNÉES}

Ces aspects soulignent encore une fois qu'une enquête quantitative réussie est le fruit d'une stratégie planifiée visant à obtenir l'adhésion, c'est-à-dire un accord conscient et éclairé sur les buts de l'enquête, d'une population représentative afin de pouvoir recueillir des données fiables. Convaincre une population n'est pas simple. Mentionnons pour exemple le cas suisse avec ses votations qui dépassent rarement les $60 \%$ de participation ${ }^{21}$ alors même que les sujets mis au vote concernent directement le votant. À plus forte raison pour une enquête sociologique où la population sondée ne perçoit pas directement son intérêt, est-il nécessaire de la convaincre de participer. Plusieurs moyens aident à augmenter son taux de participation.

Nous avons évoqué dans les paragraphes précédents le problème de populations plus difficiles à atteindre que d'autres. De ce fait, plusieurs biais peuvent gêner le sondage, d'une part du fait d'une possible surreprésentation d'une partie de la population, particulièrement volontaire et désireuse d'y contribuer, et d'autre part du fait d'une sous-représentation d'une population difficile à atteindre ou peu encline à y participer. Avant même de débuter la collecte, il est donc premièrement nécessaire d'identifier les strates de la population sur laquelle il faut concentrer une énergie particulière. On peut ainsi travailler en amont, comme cela a été fait à Fribourg, afin d'obtenir l'accord des cohortes moins volontaires au premier abord, de leur utilité-pour l'enquête. Mais plus généralement, le succès d'une enquête se joue sur son annonce. Pour cela, tous les canaux d'information doivent être mobilisés pour expliquer les enjeux de l'enquête. Il s'agit, au-delà de son annonce, d'expliquer sa pertinence pour la science et tout autant pour la population des répondants. Dans l'enquête de Fribourg, il était important de souligner que nous cherchions à connaître l'avis des membres sur les messes pour pouvoir remodeler les horaires et offrir des célébrations plus proches des besoins des fidèles. Il fallait informer afin que les rumeurs ne prennent le dessus ou que l'enquête soit interprétée comme

\footnotetext{
${ }^{21}$ En février 2016, le taux de participation est monté à 63\%, seuil qui n'avait pas été atteint depuis vingt ans.
} 
un prétexte pour obtenir des chiffres en vue d'une fusion de paroisses, sujet sensible certes, mais qui ne faisait pas partie des intentions des auteurs. L'annonce permet ainsi de clarifier les tenants et les aboutissants pour que la participation soit libre et consciente des enjeux soulevés par l'enquête.

Tableau 5 : Points importants pour annoncer l'enquête

\begin{tabular}{|l|l|}
\hline Annonces/information/courrier & Pratiquement \\
\hline Présenter l'objet d'étude & $\begin{array}{l}\text { Montrer ce que l'enquête cherche à savoir et } \\
\text { comment elle s'efforce de l'obtenir }\end{array}$ \\
\hline Sensibiliser à l'intérêt de la collecte & $\begin{array}{l}\text { Montrer l'intérêt du sujet de recherche et } \\
\text { l'importance de la population pour éclairer } \\
\text { ce sujet }\end{array}$ \\
\hline Rassurer le répondant & $\begin{array}{l}\text { Montrer que l'enquête est simple à effectuer } \\
\text { et que les aspects légaux de protection des } \\
\text { données et de la vie privée sont respectés }\end{array}$ \\
\hline Mettre les formes & $\begin{array}{l}\text { Montrer que chaque réponse, chaque } \\
\text { répondant est considéré poliment et } \\
\text { dignement }\end{array}$ \\
\hline
\end{tabular}

Cet ultime tableau synthétise quatre points essentiels qu'il est important de vérifier dans les annonces de l'enquête. Présenter l'enquête en soulignant l'objet d'étude et son cadre de réalisation permet au répondant potentiel de se situer. Il faut encore lui montrer l'intérêt de cette recherche, non pas simplement du point de vue scientifique mais, de manière plus concrète, ce qu'elle peut lui apporter. Pour cela, il est utile de le rassurer sur sa participation, la facilité et la rapidité du sondage, et sur l'existence de dispositions légales qui le protègent, de façon à rendre le sondage engageant. Même si l'information est passée par plusieurs canaux, le courrier personnel offrant une présentation des buts de l'enquête augmente le taux de participation.

Nous avons cherché à apporter un éclairage sur les enjeux d'une enquête quantitative en milieu communautaire ou religieux associatif. L'analyse statistique n'a pas été abordée, sachant que souvent cette tâche est volontiers déléguée à des statisticiens, contrairement à la partie, en amont de la préparation, des questions et de la passation du sondage auprès des répondants. Une croyance populaire tenace soutient qu'il est facile de réaliser un sondage, alors qu'il serait difficile d'analyser les réponses en raison des compétences particulières que requiert la statistique. 
Nous avons souligné ici qu'une enquête quantitative est une entreprise de longue haleine et qu'il fallait au moins autant de compétences pour préparer un bon sondage que pour analyser statistiquement des résultats. L'intention première de cette contribution est de préparer les lecteurs à anticiper les écueils de l'enquête quantitative. Pour des raisons de format, nous avons particulièrement attiré l'attention d'une part sur le protocole d'enquête avec une finalité d'enquête reposant sur des hypothèses scientifiques ; d'autre part sur la fiabilité de l'enquête avec la nécessité de définir une population représentative de la réalité ; ensuite sur les méthodes d'administration d'un questionnaire ; puis sur l'échantillonnage et finalement sur la collecte des données. D'autres écueils sont à anticiper, comme la qualité des interrogations constituant le questionnaire. De plus, les milieux paroissiaux et religieux étant au centre de fortes reconfigurations, il vaut la peine de produire des données de haute qualité. Les données chiffrées sont insuffisantes à éclairer ce phénomène. Cependant une enquête menée dans les standards sociologiques ne manquera pas d'apporter des éclairages d'intérêt, qu'elle soit menée localement, nationalement ou internationalement, et qu'elle soit dirigée par des théologiens pratiques ou des sociologues.

Christophe MONNOT 